\title{
Detection of Specific Noncovalent Interaction of Peptide with DNA by MALDI-TOF
}

\author{
Shi-Zhong Luo, Yan-Mei Li, Wei Qiang, and Yu-Fen Zhao \\ The Key Laboratory for Bioorganic Phosphorus Chemistry, Ministry of Education, Department of Chemistry, \\ Tsinghua University, Beijing, China
}

\author{
Hiroshi Abe, Tadashi Nemoto, Xu-Rong Qin, and Hiroshi Nakanishi \\ Biological Information Research Center, National Institute Advanced Industry Science and Technology AIST, \\ Tsukuba, Japan
}

\begin{abstract}
Matrix-assisted laser desorption/ionization (MALDI) mass spectrometry was used to obtain spectra of peptide-DNA complexes formed by basic domain (BD15) of c-Fos protein and DNA AP-1 site (5'-TGAGTCA-3'). The noncovalent interaction between single stranded DNA and BD15 was observed and confirmed to be an ionic one between the negatively charged sugar-phosphate backbone of DNA and positively charged side chains of Arg- and lys-rich peptides as demonstrated by Vertes and coworkers [1] and Woods and coworkers [2]. But the specific noncovalent interaction between DNA AP-1 site and the dimer of BD15 was firstly detected in this paper. Various different sequence DNAs were studied and it was found that this interaction is a sequence-specific one, and AP-1 site was essential for this interaction. This specific interaction depends on the matrix. It was only observed in the ATT matrix and not in the other two matrixes (CHCA and DHBA). (J Am Soc Mass Spectrom 2004, 15, 28-31) () 2004 American Society for Mass Spectrometry
\end{abstract}

$\mathrm{T}$ The function of a biomolecule usually depends on its specific, noncovalent interactions with other molecules. For example, interactions resulting from peptides with other peptides, proteins, and oligonucleotides drive critically important cellular processes such as cell division, cell signaling, ion transport, gene transcription, and translation [3]. Interaction between nucleic acids and proteins plays an important role in numerous biochemical processes, including DNA replication, recombination, and repair. Noncovalent interactions have been studied previously, most often using electrospray ionization (ESI) mass spectrometry [4, 5]. One of the motivations of this approach is the possibility that the structure and conformation of the gas-phase ions could retain clues to biological activity. However, noncovalent complexes are often in the presence of interferants (i.e., salts, detergents, or other solubilizing agents), which suppress ESI, making MALDI a more suitable ionization method [6]. Moreover compared with ESI, MALDI was a viable alternative to investigate noncovalent interactions due to the lack of multiply charged ions [7].

Despite the new opportunities provided by MALDI,

Published online November 4, 2003

Address reprint request to Dr. Y.-M. Li, Department of Chemistry, The Key Laboratory for Bioorganic Phosphorus Chemistry, School of Life Science and Engineering, Tsinghua University, Beijing 100084, China. E-mail: liym@mail.tsinghua.edu.cn there have only been sporadic examples to study noncovalent interaction of biomolecules by the application of mass spectrometry. The detection of such complexes by MALDI is strongly dependent on the matrix and sample preparation [8,9]. Anfinsen [10] has shown that loss of biological function could occur through disruption of noncovalent bonds of the tertiary structure, and that this structure disruption could be caused by sample preparation, temperature and/or pressure changes. So it's critical to prepare the samples properly and to choose appropriate matrixes for MALDI mass analysis.

Several laboratories have reported using MALDI to observe the noncovalent interactions [11-13]. Lecchi and Pannel showed that a less acidic matrix 6-aza-2thiothymine (ATT) in the presence of ammonium citrate allowed the detection of intact double stranded DNA [14]. Biemann's and Vertes and coworkers [1] and Juhasz and Biemann [15] demonstrated the attachment between highly acidic DNA strands and basic peptides. Further work by Woods et al. detected noncovalent interaction of single and double stranded DNA with peptides $[2,16]$. But these interactions are not DNA sequence specific; the interaction between the negatively charged sugar phosphate backbone of DNA and the positively-charged side chains of Arg- and Lys-rich peptides is just an ionic interaction.

These reports prompted us to investigate the application of this technique to detect sequence-specific 
Table 1. Peptide and oligonucleotides used in the noncovalent binding experiment

\begin{tabular}{llc}
\hline Peptides/oligonucleotides & \multicolumn{1}{c}{ Sequence } & $\mathrm{Mw}$ \\
\hline \hline BD15 & IRRERNKMAAAKCRN-NH 2 & 1816.2 \\
AP-1 & 5'-TGAGTCA-3' & 2120.4 \\
AP-1' & 5'-TGACTCA-3' & 2080.4 \\
AP-1-12mer & 5'-CTATGAGTCATC-3' & 3718.4 \\
AP-1-12mer' & 5'-CTATGACTCATC-3' & 3592.4 \\
DNA1 & 5'-CGCGCG-3' & 1793.3 \\
DNA2 & 5'-CGCGAATTCGCG-3' & 3642.6 \\
DNA3 & 5'-CCAACGTTGG-3' & 3027.5 \\
DNA4 & 5'-GCGTATACGC-3' & 3027.5 \\
\hline
\end{tabular}

DNA-binding interaction with protein. AP-1 proteins (e.g., Jun, Fos) bind specific DNA sequences, termed AP-1 site (5'-TGAGTCA-3') that are present within the regulatory regions of many different genes [17, 18]. It has been identified by X-ray crystallography that AP-1 proteins dimerize to form the complexes capable of binding AP-1 site DNA elements [19]. In this paper we will present our results of the detection of not only the ionic interaction between single stranded DNA and BD15, but also the specific noncovalent interaction between DNA AP-1 site and the dimer of BD15. The sample preparation and selection of matrix will also be discussed.

\section{Materials and Methods}

\section{Peptides and DNAs}

BD15 (IRRERNKMAAAKCRN-NH ${ }_{2}$ ) was synthesized by means of the Fmoc strategy using a simultaneous multiple peptide synthesizer, Model PSSM-8 (Shimadzu, Kyoto, Japan) with PyBOP/HOBt method. Crude peptide was purified by reverse phase HPLC on an ODS column $(20 \mathrm{~mm} \times 150 \mathrm{~mm})$ heated at $40{ }^{\circ} \mathrm{C}$ with a linear gradient of 20 to $30 \%$ acetonitrile in an aqueous solution of $0.01 \mathrm{~N} \mathrm{HCl}$ over $10 \mathrm{~min}$ at a flow rate of 9.9 $\mathrm{mL} / \mathrm{min}$. The purified peptide was lyophilized and stored at $-20{ }^{\circ} \mathrm{C}$ before used. DNAs (Table 1) were purchased from Shanghai Sangon Biological Engineering Technology and Service Co. Ltd. (Shanghai, China).

\section{Matrix}

6-aza-2-thiothymine (ATT), 2,5-dihydroxybenzoic acid (DHBA) and $\alpha$-cyano-4-hydroxycinnamic acid (CHCA) were purchased from Wako (Japan). All matrixes were prepared freshly in acetonitrile $/ 8 \mathrm{mM} \mathrm{NH}_{4} \mathrm{HCO}_{3}$ (vol/ vol, 1:1) at a concentration of $10 \mathrm{mg} / \mathrm{mL}$.

\section{Sample Preparation}

The peptide and oligonucleotide stock solutions were prepared in deionized water at $100 \mu \mathrm{M}$ concentration. $10 \mu \mathrm{L}$ BD15 and $10 \mu \mathrm{L}$ oligonucleotide aliquots were mixed in a vial and incubated at room temperature. 0.5 $\mu \mathrm{L}$ of this mixture plus $0.5 \mu \mathrm{L}$ matrix were deposited on the sample slide and left to dry at room temperature.

\section{MALDI-TOF Mass Spectrometry}

MALDI-TOF mass spectra were acquired in linear negative mode on a KOMPACT MALDI IV (Shimadzu), equipped with a nitrogen laser $(337 \mathrm{~nm})$ and an acceleration energy of $20 \mathrm{kV}$ [20].

\section{Results and Discussion}

\section{Noncovalent Interaction of DNA with BD15}

DNA-protein complexes have been extensively studied using a variety of methods, including X-ray crystallography, NMR, absorption, fluorescence, and UV spectroscopy. Most of these methods, however, require large quantities of samples, which are not always available. In these cases MALDI MS can be applied as an alternative method to study the DNA-protein interactions. Considering the fact that the basic domain of c-Fos protein binds specific DNA sequences, termed AP-1 site (5'-TGAGTCA-3'), it was expected that strong interaction between basic domain (BD15) of c-Fos and certain DNA (AP-1 site) would be detected by MALDITOF MS. Mixtures of equal amounts of a solution of BD15 and a solution of AP-1 were made and examined in negative mode by MALDI. When using ATT as matrix, non-convalent binding complexes of BD15 and single-stranded, double-stranded AP-1-7mer DNA were observed (Figure 1). In order to determine if the sequence of the DNA plays a role in this interaction, several DNAs with different sequences were employed to test their ability to bind with BD15. The spectra indicated the formation of DNA and peptide complex in both cases (Table 2). The results confirm that the interaction between the negatively-charged sugar phosphate backbone of DNA and the positively-charged side chains of Arg- and Lys-rich peptides is an ionic interaction, which is in agreement with Wood's result that this interaction is not DNA sequence specific, but just an ionic interaction.

\section{Specific Noncovalent Interactions of DNA with BD15}

The results of the formation of non-convalent binding complexes between BD15 and single-stranded, doublestranded DNAs were summarized in Table 2. Further analysis of the interaction between Fos and DNA has shown that the formation of that protein-DNA complex requires a specific DNA site identical to the recognition sequence of AP-1 [21]. To explore this specific noncovalent interaction between AP-1 and BD15, AP-1-7mer was used in a noncovalent binding assay to test its specific ability to bind with BD15. In the presence of the ATT matrix, a new peak, corresponding to the binding complex of the dimer of BD15 and certain DNA AP-1 

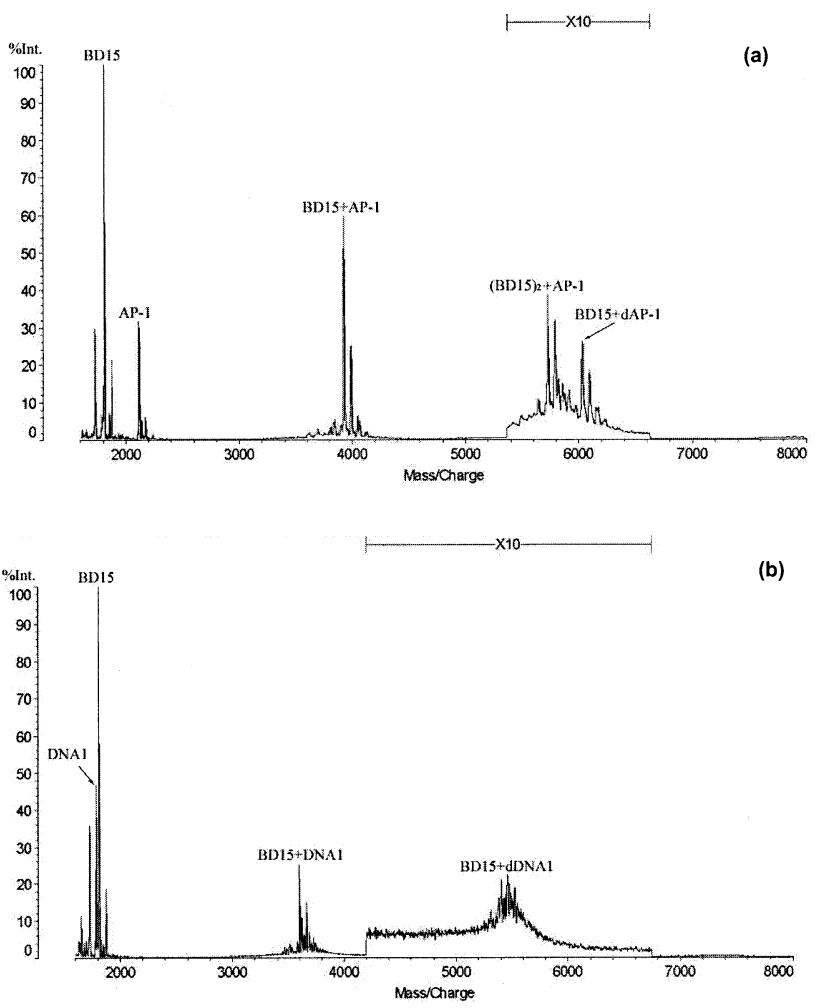

Figure 1. Linear negative MALDI mass spectra of nonconvalent binding complexes of BD15 and DNA. The matrix is ATT in $50 \%$ acetonitrile $+8 \mathrm{mM} \mathrm{NH}_{4} \mathrm{HCO}_{3}$ aqueous solution at a concentration of $10 \mathrm{mg} / \mathrm{ml}$. (a) BD15 + AP-1; (b) BD15 + DNA1.

appeared (Figure 1). In order to determine if the sequence of the DNA plays a role in this interaction, several DNA's with different sequences were employed to test their ability to bind with BD15. For the other random sequence DNA (DNA1, DNA2, DNA3, DNA4), binding complex of the dimer of BD15 and DNA was not observed (Figure 1, Figure 2, Table 2). When another AP-1 site (5'-TGACTCA-3') and longer DNA strands (AP-1-11mer) containing AP-1 site were chosen, the same phenomenon was observed (Figure 2). A reasonable explanation is that the single strands $\mathrm{BD} 15$ didn't recognized the AP-1 site and the interaction between DNA and BD15 detected by MALDI-TOF MS

Table 2. Noncovalent interactions of DNAs with BD15

\begin{tabular}{lllll}
\hline DNA & $\begin{array}{c}\text { ssDNA }{ }^{a}+ \\
\text { BD15 }\end{array}$ & dsDNA $^{\text {b }}$ & $\begin{array}{c}\text { dsDNA+ } \\
\text { BD15 }\end{array}$ & $\begin{array}{c}\text { ssDNA+ } \\
(B D 15)_{2}{ }^{c}\end{array}$ \\
\hline \hline AP-1 & Yes & No & Yes & Yes \\
AP-1 & Yes & Yes & Yes & Yes \\
AP-1-12mer & Yes & Yes & & Yes \\
AP-1-12mer' & Yes & No & & Yes \\
DNA1 & Yes & No & Yes & No \\
DNA2 & Yes & No & & No \\
DNA3 & Yes & No & & No \\
DNA4 & Yes & No & & No \\
\hline
\end{tabular}

asingle-stranded DNA.

${ }^{b}$ Double-stranded DNA.

'Dimer of BD15.

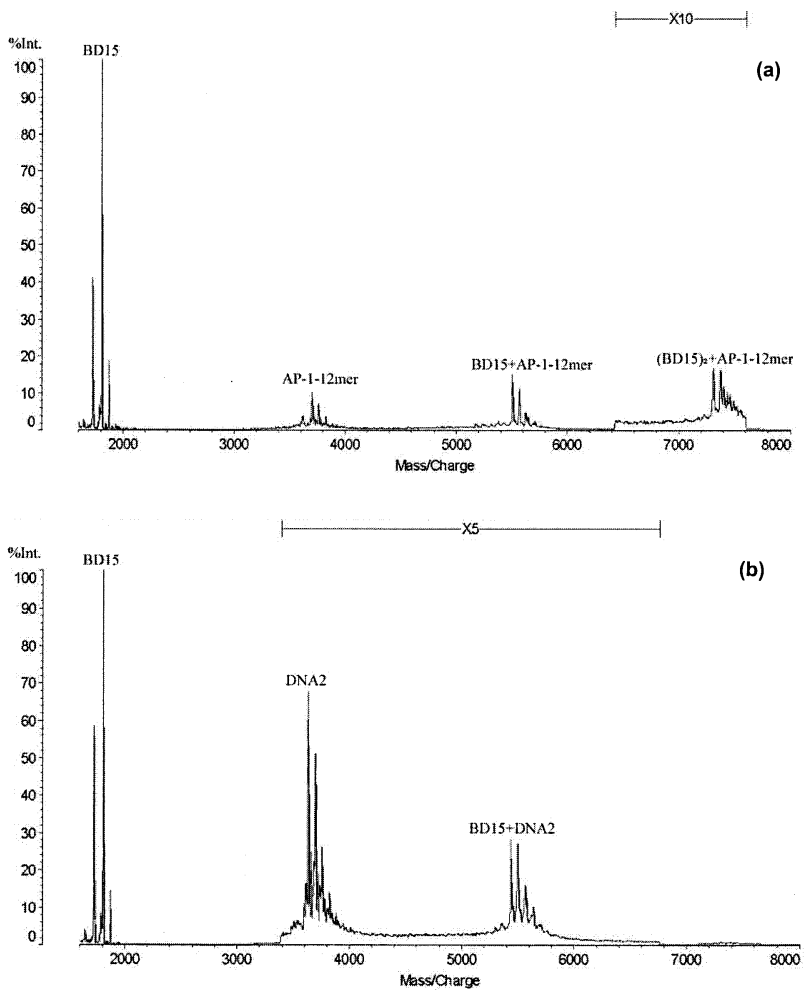

Figure 2. Linear negative MALDI mass spectra of nonconvalent binding complexes of BD15 and DNA. The matrix is ATT in $50 \%$ acetonitrile $+8 \mathrm{mM} \mathrm{NH}_{4} \mathrm{HCO}_{3}$ aqueous solution at a concentration of $10 \mathrm{mg} / \mathrm{ml}$. (a) BD15 + AP - $1-12$ mer; (b) BD15 + DNA2.

is just an ionic interaction between the negativelycharged sugar phosphate backbone of DNA and the positively-charged side chains of Arg- and Lys-rich peptides and without DNA sequence specific. But after BD15 forms a dimer, it will interact with AP-1 specifically, which is in accord with a previous report that c-Fos and c-Jun protein heterodimerize to form complexes capable of binding 5'-TGAGTCA-3' DNA elements.

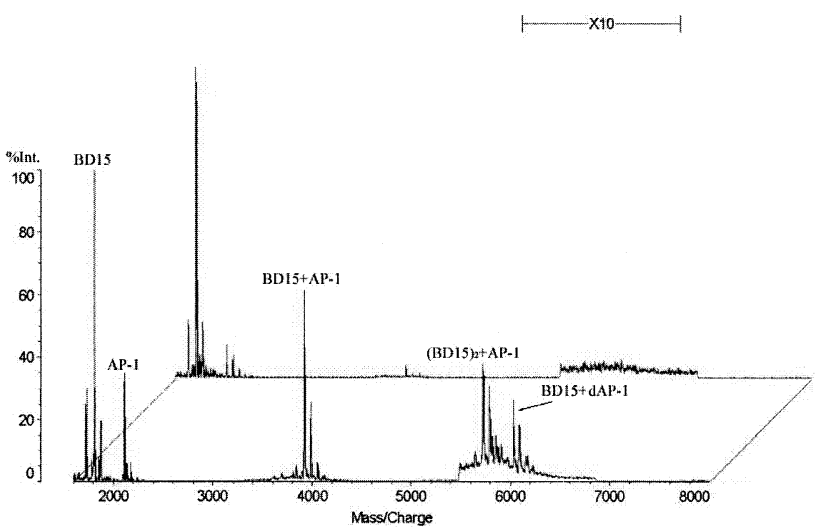

Figure 3. Linear negative MALDI mass spectra of nonconvalent binding complexes of BD15 and DNA. (a) The matrix is ATT in $50 \%$ acetonitrile $+8 \mathrm{mM} \mathrm{NH}_{4} \mathrm{HCO}_{3}$ aqueous solution at a concentration of $10 \mathrm{mg} / \mathrm{ml}$. $\mathbf{b}$ The matrix is DHBA in $50 \%$ acetonitrile + $8 \mathrm{mM} \mathrm{NH}_{4} \mathrm{HCO}_{3}$ aqueous solution at a concentration of $10 \mathrm{mg} / \mathrm{ml}$. 


\section{Role of the Matrix}

The observation and the intensity of peptide-DNA complex ions were highly dependent on matrix. To demonstrate this effect, peptides-DNA complex detection was compared in different matrixes (ATT, CHCA, and DHBA). MALDI mass spectra of the equimolar mixture of BD15 and AP-1 in DHBA and ATT matrixes are shown in Figure 3. In DHBA matrix, only BD15, DNA and noncovalent binding complexes of BD15 with DNA appeared, whereas no complexes of the dimer of BD15 and certain DNA AP-1 were observed. When CHCA was used as the matrix, no complexes were observed. Only the $\mathrm{MH}^{+}$of the free peptide and DNAs were detected. A logical explanation is that the strong acidic condition of DHBA ( $\mathrm{pH}$ 1.7) and CHCA ( $\mathrm{pH} 2.4)$ disrupted these specific noncovalent interactions $[1,2]$. Nevertheless, ATT is a good overall matrix choice for studying DNA and peptide specific noncovalent binding complex.

\section{Conclusion}

According to our study, the noncovalent interaction between single stranded DNA and BD15 was observed. Meanwhile the specific noncovalent interaction between DNA AP-1 site and the dimer of BD15 was also detected. Various different sequence DNAs were studied and it was found that this interaction is a sequencespecific one; the dimer of BD15 and AP-1 site was essential for this interaction. Compared with DHBA and CHCA, ATT is a good overall matrix choice for studying DNA and peptide specific noncovalent binding complex.

\section{Acknowledgments}

The authors thank Dr. J. Ma, Dr. M. Dahlen, and Professor Y. P. Feng for their kind help. They also thank the Chinese National
Natural Science Foundation (no. 20072023 and no. 20320130046) and Tsinghua University for financial supports.

\section{References}

1. Tang, X.; Callahan, J. H.; Zhou, P.; Vertes, A. Anal. Chem. 1995, $67,4542-4548$.

2. Lin, S.; Cotter, R. J.; Woods, A. S. Prot. Struct. Funct. Genet. 1998, 2(Suppl.), 12-21.

3. Mcbride, K.; Nemer, M. Mol. Cell. Biol. 1998, 9(18), 5073-5081.

4. Cheng, X. H.; Harms, A. C.; Goudreau, P. N.; Terwilliger, T. C.; Smith, R. D. Proc. Natl. Acad. Sci. U.S.A. 1996, 93, 7022-7027.

5. Hsieh, Y. L.; Li, Y. T.; Henion, J. D.; Ga-nem, B. Biol. J. Mass. Spectrom. 1994, 23, 272-276.

6. Woods, A. S.; Koomen, J. M.; Ruotolo, B. T.; Gillig, K. J.; Russel, D. H.; Fuhrer, K.; Gonin, M.; Egan, T. F.; Schultz, J. A. J. Am. Soc. Mass. Spectrom. 2002, 13, 166-169.

7. Krutchinsky, A. N.; Zhang, W. Z.; Chait, B. T. J. Am. Soc. Mass. Spectrom. 2000, 11, 493-504.

8. Dai, Y. Q.; Whittal, R. M.; Li, L. Anal. Chem. 1999, 71, 1087-1091.

9. Kim, Y; Hurst, G. B. Microchem. J. 2001, 71, 219-228.

10. Anfinsen, C. B. Science 1973, 181, 223-230.

11. Farmer, T. B.; Caprioli, R. M. J. Mass. Spectrom. 1998, 33, 697-704.

12. Cheng, X. H.; Morin, P. E.; Harms, A. C.; Bruce, J. E.; BenDavid, Y.; Smith, R. D. Anal. Chem. 1996, 239, 35-40.

13. Woods, A. S.; Buchsbaum, J. C.; Worrall, T. A.; Berg, M. J.; Cotter, R. J. Anal. Chem. 1995, 67, 4462-4465.

14. Lecchi, P.; Pannell, L. K. J. Am. Soc. Mass. Spectrom. 1995, 6, 972-975.

15. Juhasz, P.; Biemann, K. Proc. Natl. Acad. Sci. U.S.A. 1994, 91, 4333-4337.

16. Lin, S. H.; Long, S.; Ramirez, S. M.; Cotter, R. J.; Woods, A. S. Anal. Chem. 2000, 72, 2635-2640.

17. Blenis, J. Proc. Natl. Acad. Sci. U.S.A. 1993, 90, 5889-5892.

18. Chiu, R.; Boyle, W. J.; Meek, J.; Smeal, T.; Hunter, T.; Karin, M. Cell 1988, 54M, 541-552.

19. Glover, J. N. M.; Harrison, S. C. Nature 1995, 373, 257-261.

20. Yamgaki, T.; Ishizuka, Y.; Kawabata, S.; Nakanishi, H. Rapid. Commun. Mass Spectrom. 1997, 11, 527-531.

21. Franza, B. R.; Sambucetti, L. C.; Cohen, D. R.; Curran, T. Oncogene 1987, 1, 213-221. 